\title{
Nif Gene Disruption Inhibits Differentiation of Odontoblasts Responsible for Root Formation and Results in Formation of Short and Abnormal Roots in Mice
}

\author{
Joo-Cheol Park, ${ }^{*}$ Yeek Herr, ${ }^{\dagger}$ Heung-Joong Kim, ${ }^{*}$ Richard M. Gronostajski, ${ }^{\ddagger}$ and Moon-Il Cho§
}

Background: Nuclear factor I genes play an important role in the development of the brain, lung, and roots of teeth. We had reported that $N$ fic-deficient mice form normal crowns, but abnormal roots of molar teeth. However, the mechanism by which the disruption of Nfic gene causes abnormal root formation remains unknown.

Methods: To understand this mechanism, the root formation in Nfic-deficient mice was examined and compared to that of wild-type mice by morphological, immunohistochemical, and in situ hybridization analyses.

Results: Nfic-deficient mice formed normal Hertwig's epithelial root sheath (HERS) but severely disrupted odontoblast differentiation, leading to the formation of aberrant odontoblasts in the early stage of root formation. They became dissociated and polygonal in shape, lost their orientation and polarity, and did not express dentin sialophosphoprotein. The abnormal roots contained trapped aberrant odontoblasts, thereby resembling osteodentin in overall morphology. No osteoclasts were associated with abnormal roots. Further, the abnormal roots exhibited a decreased number of cementoblasts and cementum formation on the root surface.

Conclusions: The loss of Nfic did not interfere with the formation of HERS, but it caused disrupted odontoblast differentiation, which resulted in the formation of short and abnormal roots, and decreased cementum. This finding suggests that root dentin is required for normal cementum formation. Therefore, Nfic may be a key regulator of root odontoblast differentiation and root formation. J Periodontol 2007;78:1795-1802.

\section{KEY WORDS}

Dentin; odontoblast.

\footnotetext{
* Department of Oral Histology and Anatomy, College of Dentistry, Chosun University, Gwang-Ju, Korea.

$\dagger$ Department of Periodontology, School of Dentistry, Kyung Hee University, Seoul, Korea.

\# Department of Biochemistry and the Program in Neuroscience, School of Medicine and

Biomedical Sciences, University at Buffalo, State University of New York at Buffalo, Buffalo, NY.

$\S$ Department of Oral Biology, School of Dental Medicine, University at Buffalo, State University of New York at Buffalo.
}

$\longrightarrow$ uring tooth development, neural crest-derived ectomesenchymal (EM) cells proliferate and form dental papilla with the ability to differentiate into odontoblasts through epithelial-mesenchymal interactions. ${ }^{1-3}$ The inner enamel epithelial cells/preameloblasts induce the differentiation of dental papilla cells into odontoblasts during crown formation, whereas Hertwig's epithelial root sheath (HERS) cells induce their differentiation into odontoblasts during root formation. ${ }^{4}$ Odontoblasts are responsible for the formation of crown and root dentin, which is the major structural component of the teeth. However, the mechanism responsible for root dentin and, thus, root formation is not clearly understood.

The nuclear factor I (NFI) family of transcription proteins consists of four members: NFI-A, NFI-B, NFI-C and NFI-X. They are expressed by four highly conserved genes (Nfia, Nfib, Nfic, and $N f i x)$ in mammals. All of the NFI proteins bind to the same DNA consensus sequence with similar apparent affinities. ${ }^{5}$ NFI proteins seem to have unique celltype-specific transcriptional modulation properties, supporting unique functions for each gene in development. For example, disruption of the Nfia gene primarily 
causes brain development defects, ${ }^{6,7}$ whereas loss of Nfib interferes with prenatal brain and lung development, indicating crucial roles in development of the brain and lung. 8,9 An additional example is related to the unique role of the Nfic gene in root formation that is expressed primarily in odontoblasts, but not in preodontoblasts and EM cells. ${ }^{9}$ When we disrupted the Nfic gene in mice by removal of its second exon that encodes the NFI-C/CTF DNA-binding and dimerization domain, $N$ fic-deficient mice exhibited normal crown formation, but short and abnormal root formation. ${ }^{10}$ However, other tissues/organs in the body, including osteoblasts and ameloblasts that are responsible for bone and enamel formation, respectively, appeared to be normal. In the present report, we describe the mechanism by which disturbance of the Nfic gene causes short and abnormal root formation. We found that Nfic-deficient mice formed morphologically normal HERS, but failed to differentiate normal odontoblasts in the early stage of root formation, resulting in short and abnormal root formation. This animal model provides invaluable information for understanding the molecular processes responsible for root and osteodentin formation.

\section{MATERIALS AND METHODS}

\section{Mice}

The homozygous $\mathrm{Nfic}^{-/-}$knockout (Nfic-deficient) mice were created by removal of the second exon from the Nfic gene, which encodes the NFI-C DNAbinding and dimerization domain as described previously. ${ }^{10}$ Knockout mice were backcrossed into the C57BL/6 background ${ }^{\|}$and were bred and maintained at the Laboratory Animal Facility of the University at Buffalo. All of the animals were housed in sterile microisolators and given water and autoclaved mouse chow. The Institutional Animal Care and Use Committee of the University at Buffalo approved all animal protocols used in this study.

\section{Light Microscopy}

Postnatal wild type (WT) and Nfic-deficient mice (postnatal 7 [P7], 10, 14, 21, and 28 days) were sacrificed by intraperitoneal injection of ketamine/xylazine and perfused with $4 \%$ paraformaldehyde in phosphate buffered saline (PBS). The mandibles and maxillae (P28 only) were removed, fixed for 2 additional hours in the same fixative, demineralized in 0.1 M EDTA containing 1\% paraformaldehyde, dehydrated, and processed for embedding in paraffin ${ }^{10}$ or resin mixture. ${ }^{11}$ For light microscopy, 5 - $\mu \mathrm{m}$ thick paraffin or 1- $\mu \mathrm{m}$ thick resin mesio-distal or bucco-lingual sections of the mesial roots were cut. Paraffin sections were stained with hematoxylin and eosin, whereas resin sections were stained with $1 \%$ toluidine blue in
0.1 M veronal acetate buffer. Sections were photographed using a light microscope. "

\section{Immunohistochemistry}

HERS were localized by immunohistochemical identification of keratin according to the procedures of Park et al. ${ }^{12}$ Briefly, 5 - $\mu \mathrm{m}$ thick paraffin sections were incubated for 2 hours at room temperature with rabbit anti-human keratin polyclonal antibody reacting with 56- and 64-kDa keratins. ${ }^{\text {A }}$ After washing with PBS, keratin molecules were localized using an avidinbiotin-peroxidase complex kit. **

\section{In Situ Hybridization}

To investigate the differentiation of odontoblasts and cementoblasts during root formation, the expression of dentin sialophosphoprotein (DSPP) and bone sialoprotein (BSP) mRNA was assessed by in situ hybridization using digoxigenin-labeled mouse DSPP and BSP cRNA sense and antisense cRNA probes as we described previously. ${ }^{10}$ Briefly, sections were deparaffinized, hydrated, treated for 20 minutes with proteinase $\mathrm{K}$, washed with PBS, and fixed with $4 \%$ paraformaldehyde. After washing with PBS, they were incubated with $0.2 \mathrm{~N} \mathrm{HCl}$ for 10 minutes to inhibit endogenous alkaline phosphatase and acetylated by incubation in $0.1 \mathrm{M}$ triethanolamine containing $0.25 \%$ acetic anhydride. Hybridization was performed overnight at $55^{\circ} \mathrm{C}$ in hybridization solution $(50 \%$ deionized formamide, $10 \%$ dextran sulfate, $1 \times$ Dehart solution, $4 \times$ saline sodium-citrate (SSC) $[1 \times$ SSC is $0.15 \mathrm{M} \mathrm{NaCl}$ plus $0.015 \mathrm{M}$ sodium citrate], $10 \mathrm{mM}$ dithiothreitol, $1 \mathrm{mg}$ of yeast $\mathrm{tRNA} / \mathrm{ml}$, and $1 \mathrm{mg}$ of salmon sperm DNA/ml) containing the digoxigenin uracil triphosphate-labeled DSPP or BSP cRNA sense or antisense probes. The probes were synthesized from the plasmids containing 434-base pair (bp) BSP and 543-bp DSPP sequences using the digoxigenin-labeled RNA labeling kit. ${ }^{\dagger \dagger}$ After washing for 30 minutes with $2 \times$ SSC- $50 \%$ formamide and 10 minutes each in $2 \times$ SSC and $0.2 \times$ SSC, sections were incubated first with blocking solution to remove nonspecific probe binding and then with blocking solution containing 1:500 sheep anti-DIG-alkaline phosphate Fab fragments. DSPP and BSP mRNAs were detected by incubation of the sections with substrates until color developed. Sections were counterstained with methyl green.

\section{Measurement of Root Length}

To measure root length, eight P21 and eight P28 mice (four each of WT and Nfic-deficient mice) were used. Although they were in different stages of tooth eruption,

\footnotetext{
The Jackson Laboratory, Bar Harbor, ME.

If Axiophot, Carl Zeiss, Thornwood, NY.

\# DAKO, Carpinteria, CA.

** Vector Laboratory, Burlingame, CA.

$\dagger \dagger$ Boehringer Mannheim, Mannheim, Germany.
} 
the formation of the mesial roots of the first mandibular molars was nearly or completely finished in WT mice, thereby demonstrating a comparable root length. One mesial root, which was cut through the midline, was selected from each mouse to be photographed, printed, and measured on micrographs. Data were analyzed for statistical significance using the non-parametric Mann-Whitney test.

\section{RESULTS}

To understand the mechanism for aberrant root formation in Nfic-deficient mice, our study focused on HERS formation and odontoblast differentiation in the early stage of root formation.

\section{HERS Formation (P7)}

It is well known that HERS induces the differentiation of EM cells into odontoblasts responsible for root dentin formation. To determine whether the failure of HERS formation in the early stage of root formation caused short and abnormal roots in Nfic-deficient mice, the formation and morphology of HERS were examined. Upon completion of crown formation, WT and Nfic-deficient mice exhibited normal HERS formation (Figs. 1A and 1B). It was composed of a bilayer of inner and outer epithelial cells and was identified clearly after immunohistochemical localization of keratin filaments (Figs. 1B and 1C). EM cells were located along the pulp side of HERS (Fig. 1A).

\section{Early (P10 to 14) Stage of Root Formation}

Next, to investigate if Nfic gene disruption caused aberrant odontoblast differentiation in Nfic-deficient mice, we examined odontoblast differentiation in the early stage of root formation. At P10, WT and Nficdeficient mice had short roots. The pulp side of a newly formed root of a WT mouse was covered with elongated and well-organized odontoblasts and shorter preodontoblasts (Fig. 2A), whereas that of an $N$ fic-deficient mouse was covered with polygonal, disorganized, and abnormal odontoblasts (Fig. 2B). Further, the abnormal root contained trapped cells (Fig. 2B). However, WT and Nfic-deficient mice had normal HERS at the apical end of the developing roots (Figs. 2A and 2B).

At P14, the root of a WT mouse was long and well developed (Fig. 3A), whereas that of an Nfic-deficient mouse was short and abnormal (Fig. 3C). In WT mice, the proximal portion of the root was covered with elongated odontoblasts with a highly polarized distribution of cellular organelles (Fig. 3A). They were attached to each other at their distal extremities by terminal webs (Fig. 3B). The nuclei were located in the pulpal end of the cell bodies, whereas the Golgi complexes were situated between the nuclei and the dentin surface (Fig. 3B). The apical region of the developing root was covered with short, columnar preodontoblasts
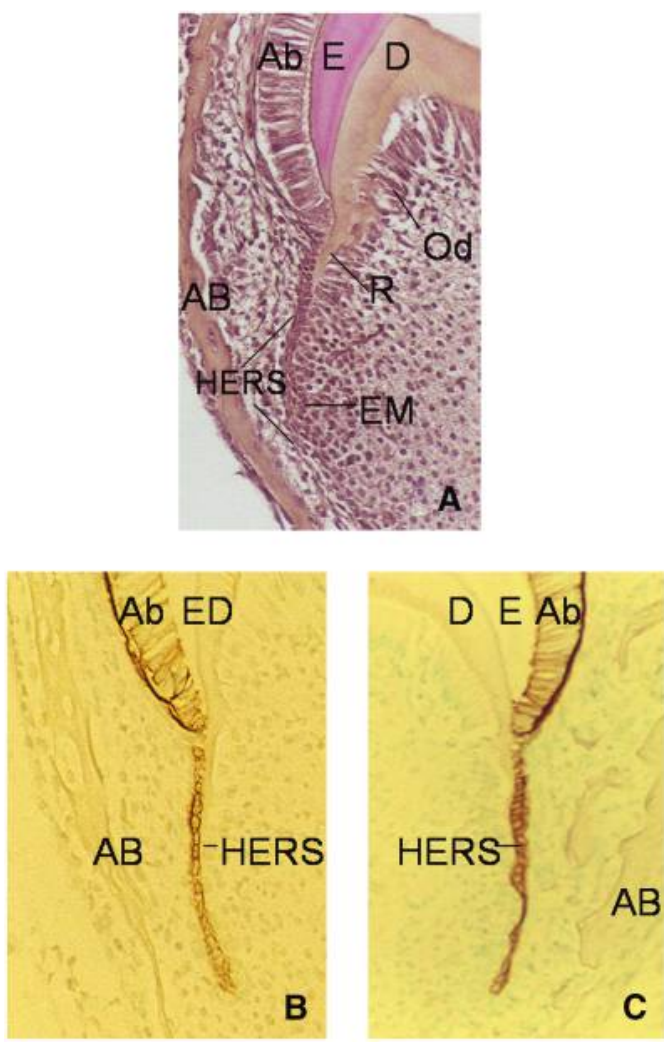

Figure I.

Light micrographs showing the early stage of first mandibular molar root formation and HERS in P7 Nfic-deficient (A and $\boldsymbol{C}$ ) and WT mice (B). Note the presence of a newly formed, short, and normal root (R) and HERS at the apical end of the root from an Nfic-deficient mouse in A. HERS in WT (B) and Nfic-deficient (C) mice demonstrate normal morphology and keratin expression by immunohistochemistry. $A b=$ ameloblasts; $A B=$ alveolar bone; $D=$ dentin; $E=$ enamel; Od = odontoblasts; $E M=$ ectomesenchymal cells. (Hematoxylin and eosin, A; original magnification $\times 180$.)

that were continuous with polygonal EM cells located along the HERS (Fig. 3B). In contrast, the short and abnormal root of an Nfic-deficient mouse was covered with polygonal aberrant odontoblasts, without any cellular polarity, that were continuous with polygonal EM cells in association with HERS (Fig. 3C). Normal HERS remained at the apical end of roots of WT and Nfic-deficient mice, although the latter appeared to have longer HERS compared to WT (Figs. 3A and $3 \mathrm{C})$. The most significant finding was the appearance of aberrant odontoblasts in the crown where the root began to form (Fig. 3D). In this region, highly polarized odontoblasts exhibited a reversed orientation. Unlike normal odontoblasts, their nuclei were located adjacent to the dentin surface. Uniquely, abnormal roots contained trapped cells, and their surfaces were covered with polygonal cells. The changes that occurred during the early stage of root formation in Nfic-deficient mice led to the formation of short and abnormal roots that resemble osteodentin. 

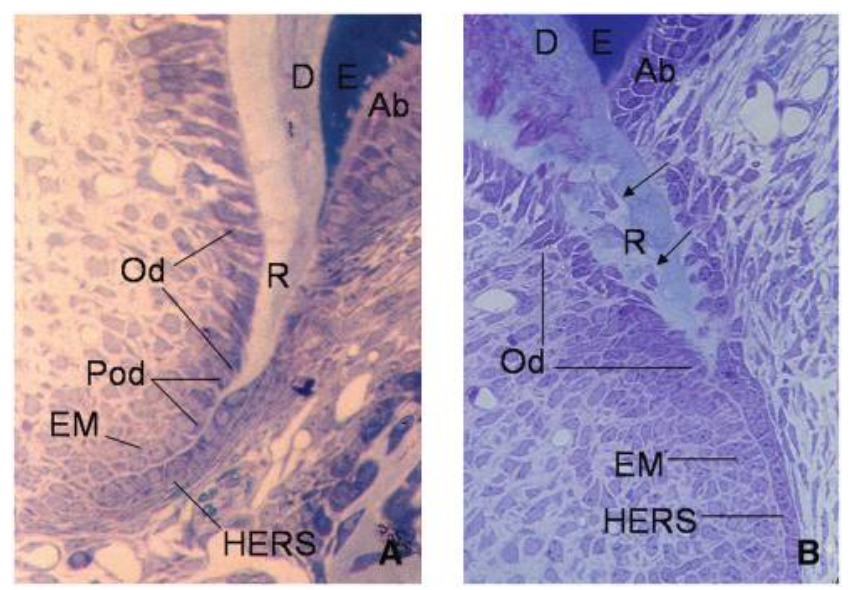

\section{Figure 2.}

Light micrographs showing developing first mandibular molar roots of P I 0 WT (A) and Nfic-deficient (B) mice. At this stage, WT (A) and mutant (B) mice have relatively short roots (R). A) The proximal portion of the root is covered with elongated and well-organized odontoblasts (Od), whereas the distal portion is covered with shorter preodontoblasts (Pod). The preodontoblasts are continuous with ectomesenchymal cells (EM) in association with HERS at the apical end of the root. B) The abnormal root of an Nfic-deficient mouse is covered with polygonal, disorganized, and aberrant odontoblasts (Od). Further, the root contains trapped cells (arrows). WT (A) and Nficdeficient (B) mice show normal HERS that is associated with EM cells. $A b=$ ameloblasts; $D=$ dentin; $E=$ enamel. (Toluidine blue, $B$; original magnification $\times 230$.)

Odontoblasts in the crown and root of a WT molar strongly expressed DSPP mRNA, whereas preodontoblasts and EM cells did not (Fig. 4A). Conversely, the odontoblasts in the crown of an Nfic-deficient molar highly expressed DSPP mRNA, but aberrant odontoblasts on the abnormal root surface failed to do so (Fig. 4B), suggesting that the abnormal roots of Nficdeficient mice may not contain DSPP.

In WT mice, the developing roots had numerous cementoblasts expressing BSP mRNA (Fig. 5A) and small-sized epithelial rests (ERs) that stained positively for keratin along the root surface (Fig. 5C). Conversely, the abnormal roots of $\mathrm{N}$ fic-deficient mice had a few cementoblasts (Fig. 5B) and large-sized aggregated ERs adjacent to their root surface (Fig. 5D).

\section{Fully Erupted Molars (P28)}

The first maxillary molars of WT and Nfic-deficient mice were fully erupted and had normal crowns. HERS was absent in both animals (Figs. 6 and 7A). In WT mice, the molar had long and fully developed roots that were housed in the alveolar bone and the periodontal ligament (PDL) between the root and alveolar bone (Fig. 6). Nfic-deficient mice had fully erupted crowns and short and abnormal roots that contain trapped cells. Noticeably, the abnormal roots had the apical foramens with a large diameter (Fig.
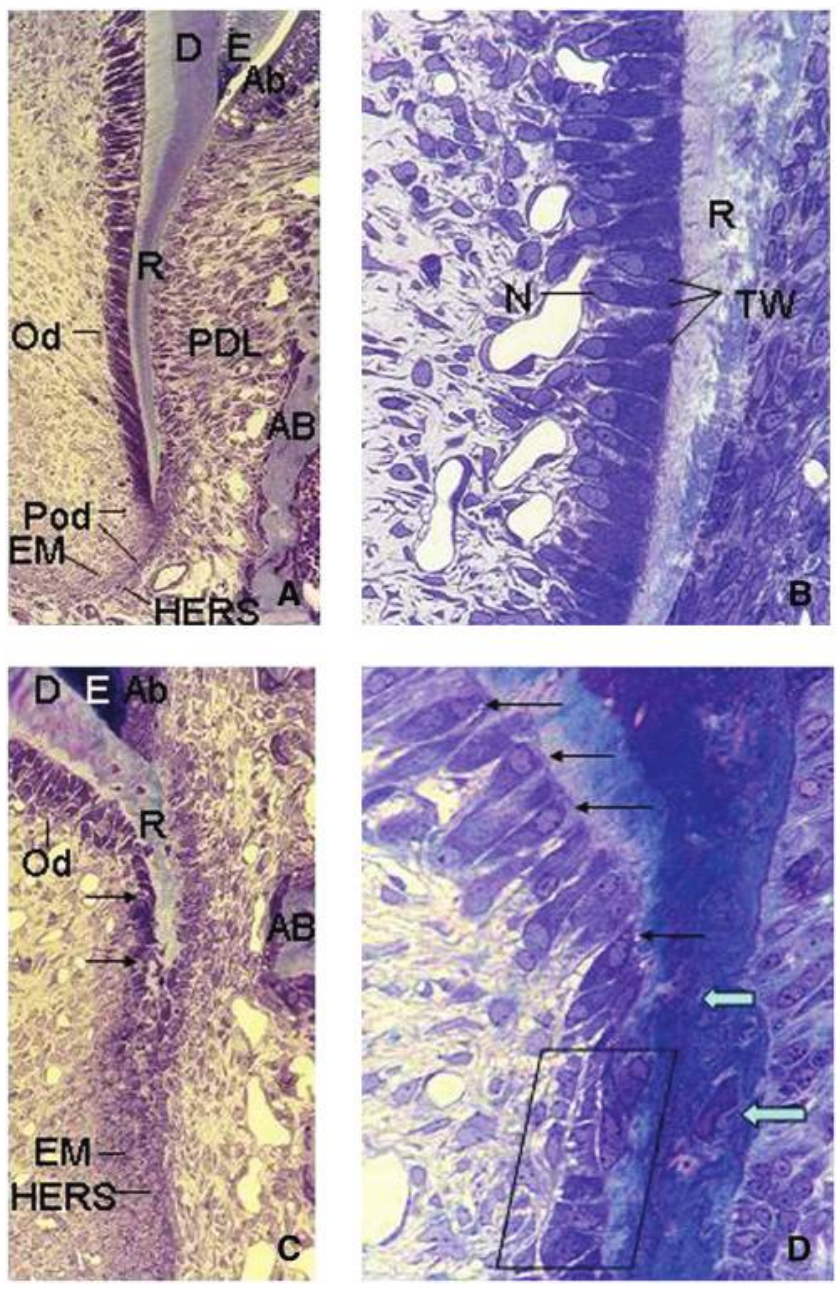

Figure 3.

Low- (A and $\mathbf{C})$ and high-magnification (B and $\mathbf{D})$ light micrographs showing the developing first mandibular molar roots from PI 4 WT ( $A$ and $B$ ) and Nfic-deficient ( $C$ and D) mice. A) The pulp side of the root from a WT mouse is covered with elongated odontoblasts (Od) and preodontoblasts (Pod). Ectomesenchymal cells (EM) are located close to HERS at the apical end of the root. B) Odontoblasts are highly polarized and well oriented. They are connected to each other at the distal end of cell bodies via the terminal webs (TW) and contain the nuclei ( $N$ ) at the pulpal ends. C) The root (R) of an Nfic-deficient mouse is short and abnormal. It is covered with disorganized, polygonal, and aberrant odontoblasts (arrows). HERS remain at the apical end of the root. D) The odontoblasts in the junction between the crown and root are elongated and highly polarized, but some show a reversed cell orientation (black arrows). Note the nuclei close to the dentin in these disoriented odontoblasts. The root contains trapped cells (thick arrows) and is covered with polygonal cells (rectangular area). $A b=$ ameloblasts; $A B=$ alveolar bone; $D=$ dentin; $E=$ enamel; $P D L=$ periodontal ligament. (Toluidine blue, $B$ and $D$; original magnification: $A$ and $C, \times 130 ; B, \times 450 ; D, \times 467$.)

7A) and were covered with numerous polygonal cells of an unknown origin (Fig. 7B). The roots were not associated with odontoclasts (Fig. 7B), suggesting that short roots of Nfic-deficient mice did not result from root resorption by odontoclasts. The pulp contained 

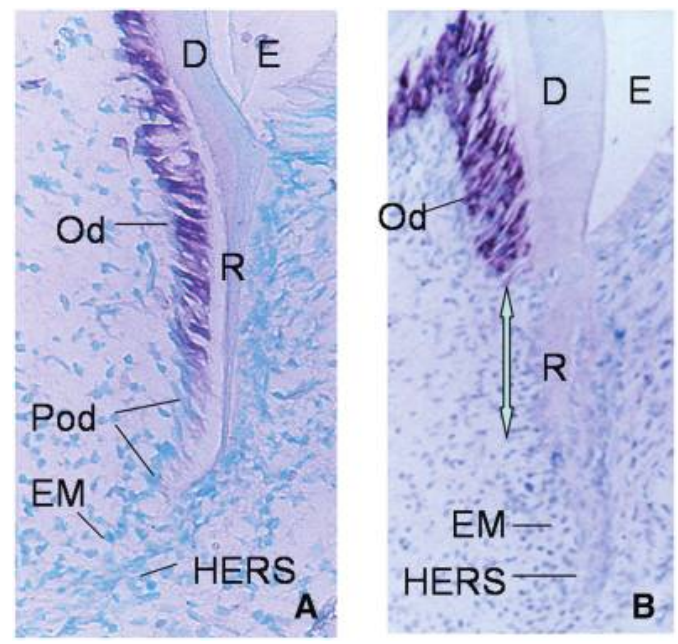

Figure 4.

In situ hybridization analysis of DSPP $m R N A$ expression in odontoblasts in the developing first mandibular molar roots from PI 4 WT (A) and Nfic-deficient (B) mice. A) The odontoblasts (Od) in the crown and root (R) of a WT mouse show strong expression of DSPP mRNA, whereas preodontoblasts (Pod) and ectomesenchymal cells (EM) do not. B) In the mutant mouse, the odontoblasts (Od) in the crown show strong expression of DSPP mRNA, but the aberrant odontoblasts (arrow) on the abnormal root surface $(R)$ do not. (Original magnifi cation: $A, \times 230 ; B, \times 340$.) $D=$ dentin; $E=$ enamel.

numerous blood vessels (Figs. 7A and 7B). No noticeable PDL was present between the abnormal roots and the alveolar bone (Fig. 7B).

\section{Measurement of Root Length}

Nfic-deficient mice had significantly shorter mesial roots of the mandibular molars $(P<0.05)$ compared to WT mice. The average root length for WT and Nfic-deficient mice was $0.83 \pm 0.06 \mathrm{~mm}$ and 0.19 $\mathrm{mm} \pm 0.08 \mathrm{~mm}$ respectively (statistically significant, Mann-Whitney test, $P<0.05)$.

\section{DISCUSSION}

Nfic-deficient mice developed normal crowns but abnormal roots on the molar teeth as a result of aberrant odontoblast differentiation during root formation. The mechanism for this differential odontoblast differentiation seen in the molar teeth is unknown. It is well known that the crown is formed during the embryonic stage, whereas the root is formed postnatally. Thus, we speculate that the function of $\mathrm{Nfic}$ in the postnatal stages of tooth development is for root odontoblast differentiation and dentin formation during molar development, and it does not have a role in crown formation. However, we do not exclude the possible involvement of other transcription factors or signaling pathways that may define the differences in gene activities between the crown and root.
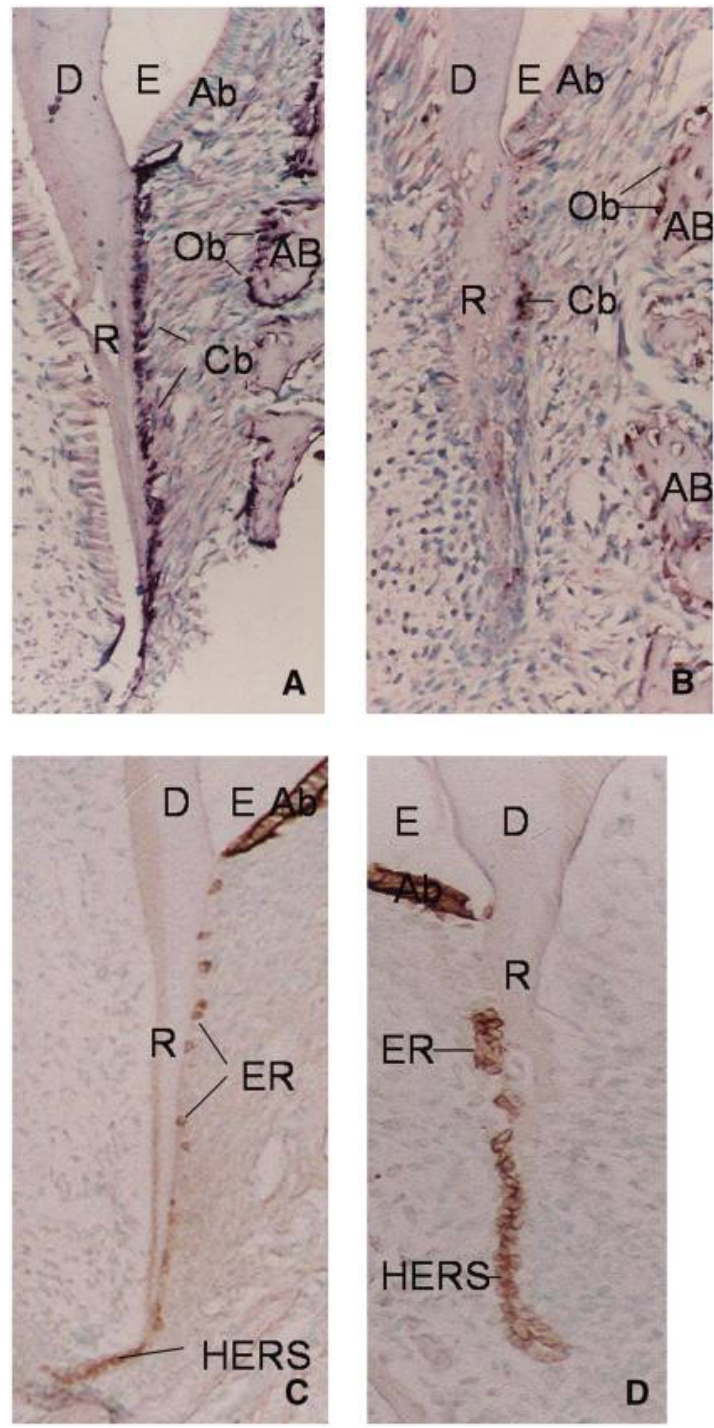

Figure 5.

In situ hybridization analysis of BSP mRNA expression (A and $\mathbf{B})$ in cementoblasts and osteoblasts and immunohistochemical localization of keratin (C and $\mathbf{D})$ in epithelial rests and HERS in the developing first mandibular molar roots from PI 4 WT (A and C) and Nficdeficient ( $B$ and D) mice. A) Note the numerous cementoblasts (Cb) along the root surface $(R)$ and osteoblasts $(\mathrm{Ob})$ on the alveolar bone $(A B)$ surface that express BSP mRNA. B) Note a few cementoblasts on the root $(R)$ surface, but many osteoblasts $(\mathrm{Ob})$ on the alveolar bone (AB) surface that express BSP mRNA. C) Note the small groups of epithelial rests (ER) along the root surface and short HERS at the apical end of the developing root (R) from a WT mouse. D) Note a large group of aggregated epithelial rests (ER) on the root surface and longer HERS at the end of the developing root $(R)$ from a mutant mouse. (Original magnification: A, $\times 270 ; B, \times 380 ; C, \times 260$; D, ×300.) $A b=$ ameloblasts; $D=$ dentin; $E=$ enamel.

Tissue recombination studies ${ }^{13}$ have demonstrated that teeth develop through the epithelial-mesenchymal interaction between the dental epithelium and cranial neural EM. Upon completion of crown formation, the inner and outer enamel epithelial cells proliferate 


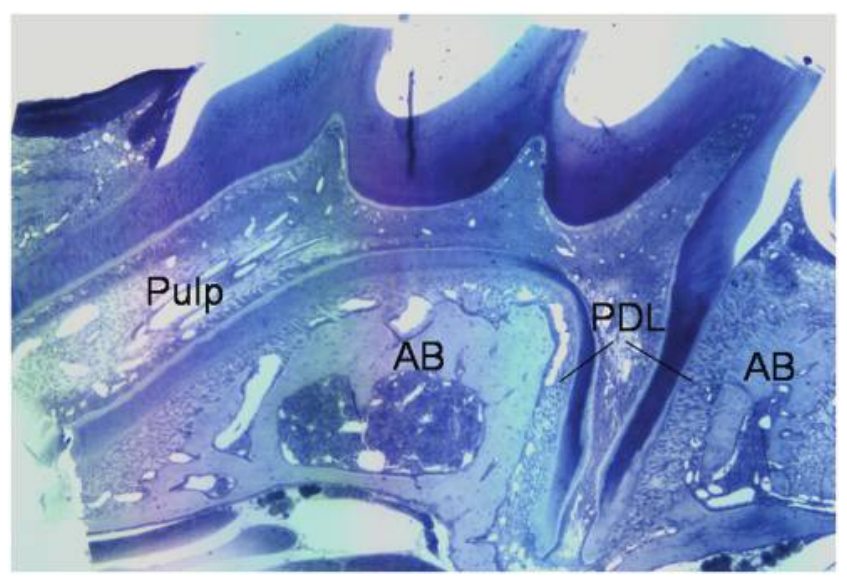

Figure 6.

Light micrograph of the first maxillary molar from a P28 WT mouse. Note the long and fully developed first maxillary roots that are anchored to the alveolar bone (AB) through the PDL. (Toluidine blue; original magnification $\times 45$.)
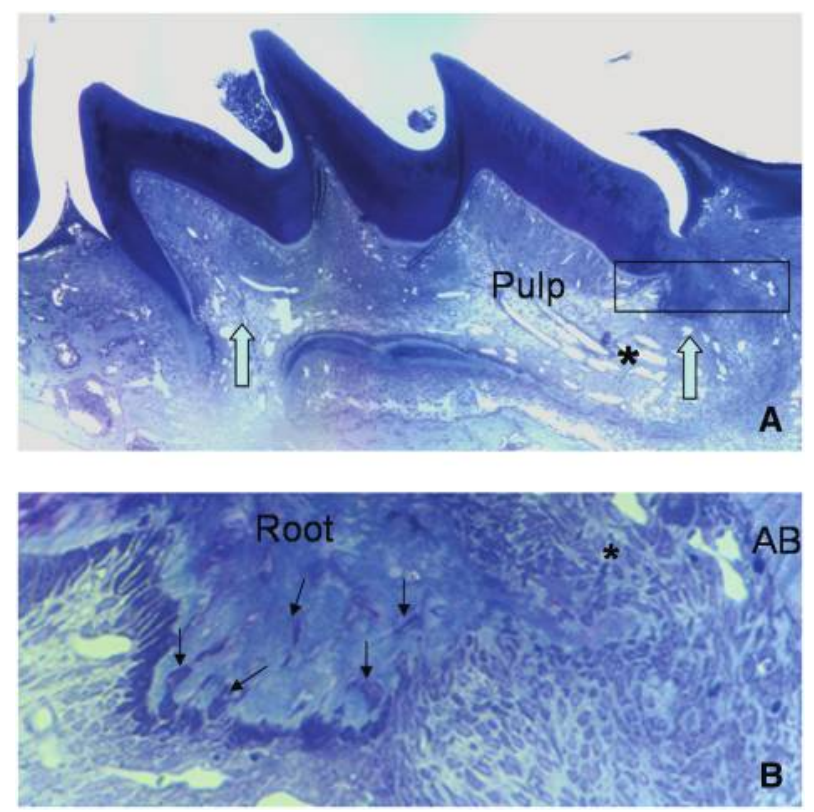

Figure 7.

Light micrographs of the first maxillary molar from a P28 Nficdeficient mouse. A) The tooth has a normal crown, but extremely short roots with widely opened apical foramens (arrows) and many blood vessels (*) in the pulp. B) Magnification of the rectangular area in A. Note $a$ short root that contains trapped cells (arrows) and dense connective tissue (*) between it and the alveolar bone (AB); no noticeable PDL is present. (Toluidine blue staining; original magnification: $A, \times 45 ; B, \times 180$.)

apically and form HERS. ${ }^{14,15}$ It is generally agreed that HERS has a key role in determining the shape of the root and in the induction of EM differentiation into odontoblasts responsible for root dentin forma- tion. The formation of morphologically normal HERS in Nfic-deficient mice soon after crown formation strongly suggests that the aberrant odontoblast differentiation observed in Nfic-deficient mice may not be the result of defects in HERS. However, we do not rule out possible functional defects of HERS at a molecular level that are due to the loss of Nfic. This may include a failure in the production of growth factors or signaling molecules necessary for normal odontoblast differentiation by HERS. However, our working model is that the loss of the Nfic gene is directly responsible for aberrant cell-autonomous odontoblast differentiation in Nfic-deficient mice.

As EM cells differentiate into preodontoblasts and eventually odontoblasts, they become elongated and highly polarized. They have the nuclei at the base of cells, the Golgi complex at the supranuclear region, and rough endoplasmic reticulum at the periphery of cell bodies. As preodontoblasts differentiate into odontoblasts, they are joined and attached at their distal end of cell bodies by well-developed terminal webs of cytoskeletal actins. ${ }^{16-18}$ This junctional complex is responsible for the alignment of odontoblasts as a single layer of cells functioning as a unit, maintaining a uniformly even dentin surface, and preventing their entrapment in the predentin. ${ }^{19,20}$ Therefore, these junctional complexes seem to play a crucial role in the formation and maintenance of smooth-surfaced predentin and dentin. More importantly, unlike osteocytes and chondrocytes, odontoblasts are not trapped in mineralized tissue (osteodentin) and are always located on the pulpal surface of the dentin. The present study revealed that $N$ fic-deficient mice have aberrant odontoblasts that were polygonal in shape. In addition, they lost their cellular polarity, orientation, and arrangement as a sheet of cells and, thus, were very disorganized. Further, they were trapped in dentinlike mineralized tissue that was formed by these aberrant odontoblasts. One of the most striking morphological changes observed in these cells was the absence of intercellular junction complexes, known as the terminal web. Based on these morphological observations, we speculate that disruption of the Nfic gene may cause dissociation of odontoblasts by interfering with the formation of intercellular junctions that contribute to aberrant odontoblast differentiation and abnormal dentin. The first sign of loss of intercellular junctions between aberrant odontoblasts in Nfic-deficient mice was observed in the junction between the crown and the newly forming root. Here, some of the highly polarized odontoblasts revealed the loss of their cellular orientation, although HERS were formed and were present at the apical end of the crown. Unlike the WT odontoblasts, they have their nuclei close to, and Golgi complex away from, the predentin. As the formation of roots began, EMcells lost their orientation, 
polarity, and attachment/connection with neighboring cells. Consequently, they were dissociated, and some of them were trapped in an abnormal mineralized tissue that was formed by the aberrant cells.

As EM cells differentiate into preodontoblasts, they synthesize and secrete types I and III collagen, osteopontin, and dentin matrix protein- $1 .{ }^{21}$ Further, when preodontoblasts differentiate into odontoblasts, they actively synthesize and deposit DSPP, the odontoblast/dentin marker protein. ${ }^{21}$ With the deposition of dentin matrix, the odontoblast cell bodies move away from the predentin/dentin, leaving the odontoblastic processes embedded within dentinal tubules in the predentin/dentin. Odontoblasts are responsible for the formation and maintenance of the predentin and dentin. The inability of aberrant odontoblasts to express DSPP indicates that EM cells failed to differentiate into odontoblasts after disruption of the Nfic gene. Based on these findings, we postulate that NFI-C transcriptional factor may be the key regulator for root odontoblast differentiation and root formation.

Nfic-deficient mice seem to offer an invaluable animal model to determine the mechanism for osteodentin formation during dentin repair. When the odontoblastic layer has been destroyed by deep caries, cells in the underlying pulp migrate to this site, differentiate into odontoblast-like cells, ${ }^{22}$ and form osteodentin that shares morphological similarities with the abnormal roots found in Nfic-deficient mice. These cells had no odontoblastic processes and failed to form intercellular junctions and associated terminal webs. The lack of intercellular junctions resulted in dissociation of the round osteoblast-like cells and loss of their cellular polarity, orientation, and organization as a sheet of cells. As a result, these cells were trapped in the mineralized tissues, resembling osteocytes. Thus, these cells deposited an irregular and disorganized dentin-like mineralized tissue in which cells become trapped and the matrix lacks dentinal tubules. We postulate that osteodentin formation during dentin repair may be the result of the involvement of pulp cells that have a silent Nfic gene during dentin repair.

We previously proposed that during normal root formation, chemoattractant substances, which may be derived from the newly formed predentin/dentin of roots, may cause directed cell migration of dental follicle cells toward the predentin/dentin surface to form cementum. ${ }^{11}$ As these cells migrate, they differentiate into precementoblasts and actively invade and push away into the epithelial sheet. The dissociated cells of the HERS remain as epithelial rests in the PDL. Upon arrival of precementoblasts on the dentin surface, they differentiate into cementoblasts and form acellular cementum on the root dentin surface. ${ }^{11}$ In the present study, we found large groups of cells of the HERS and a small number of cementoblasts on the short and abnormal roots in $\mathrm{Nfic}^{-/-}$mice. These findings support the notion that normal dentin formation is required for the formation of normal acellular cementum, epithelial rests, and PDL.

Short-root anomaly has been reported, generally in incisors and premolars. ${ }^{23-25}$ The prevalence in healthy, young Finnish adults and European children ( $\leq 14$ years) was $1.3 \%^{26}$ and $2.4 \%$ to $2.7 \%,{ }^{27,28}$ respectively. Individuals without recognized syndromes had short-root anomaly, ${ }^{29,30}$ and some seem to be inherited in an autosomal-dominant transmission. ${ }^{31}$ The etiology of short-root anomaly remains unknown. It is of great interest to examine whether short-root anomaly, particularly in those without any syndromes, is caused by the defect or functional loss of the Nfic gene as seen in Nfic-deficient mice.

\section{CONCLUSIONS}

The Nfic gene may be the key regulator for root odontoblast differentiation and root formation. Further, Nfic-deficient mice are an invaluable and unique experimental animal model that allows us to determine the apparent central role of NFI-C in odontoblast differentiation, particularly during postnatal root dentin and osteodentin formation.

\section{ACKNOWLEDGMENTS}

This work was supported, in part, by the Basic Research Program of the Korea Science and Engineering Foundation (grant RO1-2006-000-10581-0) and the Interdisciplinary Research and Creative Activities Fund at the State University of New York at Buffalo (SUNY-Buffalo). The authors thank Dr. George SteelePerkins, Department of Biochemistry, SUNY-Buffalo, and Kenneth Butz, Department of Biochemistry, SUNY-Buffalo, for the initial generation and breeding of $\mathrm{N} \mathrm{fic}^{-/}$mice, respectively.

\section{REFERENCES}

1. Kollar EJ, Baird GR. The influence of the dental papilla on the development of tooth shape in embryonic mouse tooth germs. JEmbruol Exp Morphol 1969;21: $131-148$.

2. Kollar EJ, Baird GR. Tissue interactions in embryonic mouse tooth germs. II. The inductive role of the dental papilla. J Embryol Exp Morphol 1970;24:173-186

3. Kollar A. Potential errors in the interpretation of results of hearing tests (in Czech). Cesk Otolarungol 1986; 35:296-300.

4. Thomas HF, Kollar EJ. Differentiation of odontoblasts in grafted recombinants of murine epithelial root sheath and dental mesenchyme. Arch Oral Biol 1989; 34:27-35.

5. Gronostajski RM. Roles of the NFI/CTF gene family in transcription and development. Gene 2000;249:31-45.

6. das Neves L, Duchala C, Godinho F, et al. Disruption of the murine nuclear factor I-A gene (Nfia) results in perinatal lethality, hydrocephalus and agenesis of 
the corpus callosum. Proc Natl Acad Sci USA 1999;96: 11946-11951.

7. Shu T, Butz KG, Plachez C, Gronostajski RM, Richards LJ. Abnormal development of forebrain midline glia and commissural projections in Nfia knock-out mice. J Neurosci 2003;23:203-212.

8. Grunder A, Ebel TT, Mallo M, et al. Nuclear factor I-B (Nfib) deficient mice have severe lung hypoplasia. Mech Dev 2002;112:69-77.

9. Steele-Perkins G, Plachez C, Butz KG, et al. The transcription factor gene $N f i b$ is essential for both lung maturation and brain development. Mol Cell Biol 2005;25: 685-698.

10. Steele-Perkins G, Butz KG, Lyons GE, et al. Essential role for NFI-C/CTF transcription-replication factor in tooth root development. Mol Cell Biol 2003;23:10751084.

11. Cho MI, Garant PR. Ultrastructural evidence of directed cell migration during initial cementoblast differentiation in root formation. J Periodontal Res 1988;23:268276.

12. Park JC, Kim YB, Kim HJ, et al. Isolation and characterization of cultured human periodontal ligament fibroblast-specific cDNAs. Biochem Biophys Res Commun 2001;282:1145-1153.

13. Mina M, Kollar EJ. The induction of odontogenesis in non-dental mesenchyme combined with early murine mandibular arch epithelium. Arch Oral Biol 1987;32: 123-127.

14. Grant D, Bernick S. Morphodifferentiation and structure of Hertwig's root sheath in the cat. $J$ Dent Res 1971;50:1580-1588

15. Owens PD. A light microscopic study of the development of the roots of premolar teeth in dogs. Arch Oral Biol 1974; 19:525-538.

16. Holland GR. An ultrastructural survey of cat dentinal tubules. J Anat 1976;122:1-13.

17. Garant PR. The demonstration of complex gap junctions between the cells of the enamel organ with lanthanum nitrate. J Ultrastruct Res 1972;40:333-348.

18. Garant PR, Cho MI. Recent studies of odontoblast structure and function: The secretory process examined with an analog and the polyene antibiotic filipin. INSERM Symposia 1984;125:143-158.
19. Linde A, Goldberg M. Dentinogenesis. Crit Rev Oral Biol Med 1993;4:679-728.

20. Piesco NP. Histology of dentin. In: Avery JK, ed. Oral Development and Histology. New York: Thieme; 2001: 177-183.

21. Butler WT. Dentin matrix protein. Eur J Oral Sci 1998; 106:204-210.

22. Bjorndal L, Darvann T, Thylstrup A. A quantitative light microscopic study of the odontoblast and subodontoblastic reactions to active and arrested enamel caries without cavitation. Caries Res 1998;32:59-69.

23. Catala M, Zaragoza A, Estrela F, Valdemoro C. Unusual case of rootless premolar. Pediatr Dent 1995;17:127-128.

24. Apajalahti S, Arte S, Pirinen S. Short root anomaly in families and its association with other dental anomalies. Eur J Oral Sci 1999;107:97-101.

25. Saini TS, Kimmes NS, Westerman GH. Aberrant root formation: Review of root genesis and three case reports. Pediatr Dent 2004;26:261-265.

26. Apajalahti S, Holtta P, Turtola L, Pirinen S. Prevalence of short-root anomaly in healthy young adults. Acta Odontol Scand 2002;60:56-59.

27. Jakobsson R, Lind V. Variation in root length of the permanent maxillary central incisor. Scand J Dent Res 1973;81:335-338.

28. Brook AH, Holt RD. The relationship of crown length to root length in permanent maxillary central incisors. Proc Br Paedod Soc 1978;8:17-20.

29. Lerman RL, Gold R. Idiopathic short root anomaly. J Pedod 1977;1:327-333.

30. Shaw JH. Effect of nutritional factors on bones and teeth. Ann N Y Acad Sci 1955;60:733-762.

31. Apajalahti S, Arte S, Pirinen S. Short root anomaly in families and its association with other dental anomalies. Eur J Oral Sci 1999;107:97-101.

Correspondence: Dr. Joo-Cheol Park, Department of Oral Histology and Anatomy, College of Dentistry, Chosun University, 375, Seo-Sug Dong, Dong-Gu, Gwang-Ju, \#501-759, Korea. Fax: 82-62-224-3706; e-mail: jcapark@ chosun.ac.kr.

Submitted September 18, 2006; accepted for publication March 5, 2007. 